\title{
A two-level emergency control scheme against power system voltage instability
}

\author{
Bogdan Otomega ${ }^{\mathrm{a}}$, Mevludin Glavic ${ }^{\mathrm{b}}$, Thierry Van Cutsem $^{\mathrm{c}}$ \\ ${ }^{a}$ Power System Department, Politehnica University of Bucharest, 313 Spl. Independentei, 060042, Bucharest, Romania \\ ${ }^{b}$ Dept. of Electrical Engineering and Computer Science, University of Liège, Sart Tilman B37, B-4000 Liège, Belgium. \\ ${ }^{c}$ Fund for Scientific Research $\mathcal{F}$ Dept. of Elec. Eng. and Comp. Sc., University of Liège, Sart Tilman B37, B-4000 Liège, Belgium \\ (e-mail: t.vancutsem@ulg.ac.be; fax: + 3243664582$)$.
}

\begin{abstract}
A two-level adaptive control scheme against power system voltage instability is proposed, to deal with emergency conditions by acting on distribution transformers and/or by curtailing some loads. The lower level includes distributed controllers, each acting once the voltage at a monitored transmission bus settles below a threshold value. The upper level benefits from wide-area monitoring and adjusts in real-time the voltage thresholds of the local controllers. Emergency detection is based on the sign of sensitivities. The proposed scheme is robust with respect to communication failures. Its performance is illustrated through detailed simulations of a small but realistic 74-bus test system.
\end{abstract}

Keywords: Power systems, voltage instability, emergency control, distributed control, wide-area monitoring, adaptive systems, load shedding, load tap changer.

\section{Introduction}

Voltage instability of power systems is linked to the inability of the combined generation-transmission system to provide the power requested by loads [四]. In a typical voltage instability scenario, the maximum power deliverable to loads drops under the effects of a large disturbance and the limitations on reactive power generation; concurrently, the loads connected to the transmission system tend to restore their powers near the value before the disturbance. Those antagonistic effects prevent the system from regaining a state of operating equilibrium with network voltages in acceptable ranges of values [2]. Depending on the involved component dynamics and the severity of the disturbance, voltage instability can evolve in time frames of several seconds (short-term instability) or tens of seconds up to several minutes (long-term instability). In this paper, the emphasis is on long-term voltage instability, in which network voltages undergo a generally monotonic decrease after the initiating disturbance.

A typical example from simulation is shown in Fig. I (obtained with the system considered in the results section). The power system is subject to a short-circuit which is cleared by opening a transmission line. The plot shows the evolution of the voltage magnitudes at a transmission and the closest distribution bus ${ }^{m}$. After the fault is cleared, the system is subject to electromechanical oscillations (of the rotors of synchronous generators) before approaching a short-term equilibrium. Next, the system evolves in the long term under the above mentioned effect of generator reactive power limitation and load power restoration.

\footnotetext{
${ }^{1}$ all voltages are shown in per unit $(p u)$, i.e. divided by the nominal voltage of the corresponding bus
}

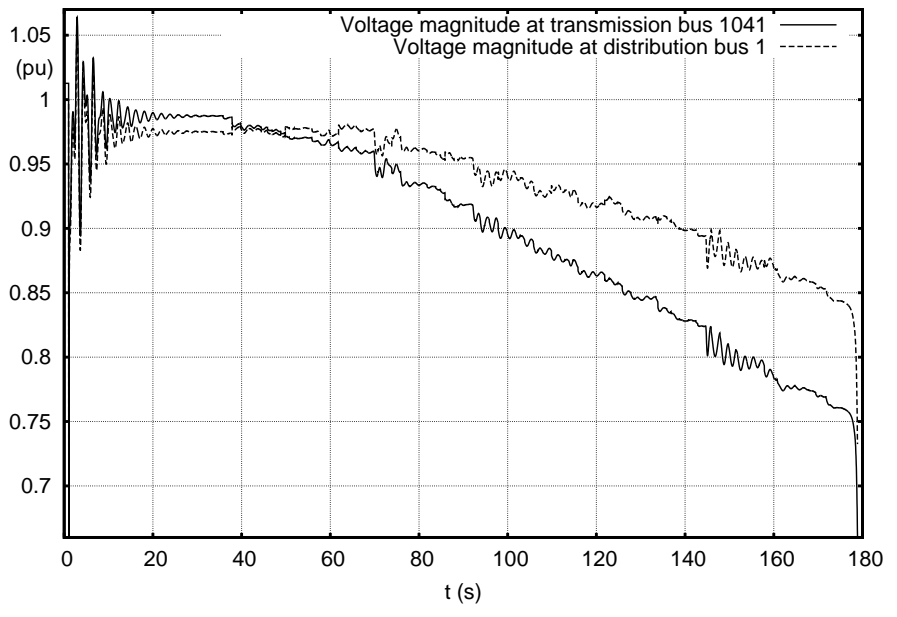

Figure 1: Example of long-term voltage instability

When pronounced, and if not controlled, voltage instability may result in voltage collapse. In the case of Fig. 1 this takes on the form of a loss of synchronism of a nearby generator, leading to the sharp final voltage drop. In practice, this will trigger a sequence of events leading most likely to a blackout. The heavy consequences of power system blackouts in terms of economical and societal costs [3, 4] motivate the improvement of control schemes to deal with voltage instability problems.

Remedies against voltage instability can be categorized into preventive actions and corrective controls.

Preventive actions consists of adjusting the operating point in order the system to be able to face each credible incident (referred to as contingency) of a predefined list. Those actions are taken in normal operating state, i.e. before the oc- 
currence of any disturbance, and involve costs to protect the system against hypothetical events. However, many voltage incidents resulted from a severe low-probability disturbance, against which it would be too expensive - if at all feasible to take preventive actions.

Corrective controls aim at acting after the actual occurrence of a disturbance. They can be broadly classified into open-loop and closed-loop. Open-loop control resorts to actions determined off-line from exhaustive simulations of postulated scenarios while closed-loop control assesses the disturbance severity through measurements, adjusts its actions correspondingly, tracks the system evolution and repeats some actions if the previous ones are not sufficient.

The dominant trend is to integrate emergency controls in System Integrity Protection Schemes (SIPS) [5] while exploiting new technological solutions such as synchronized phasor measurements [6] and fast communications as the main enablers of power system Wide Area Monitoring System (WAMS) [7]. New algorithms are needed to process the data collected in WAMS and effectively control unstable system evolutions.

This paper deals with such an algorithm. It proposes an adaptive two-level emergency control scheme aimed at driving the voltage unstable system towards a new, acceptable equilibrium [1]. The lower level consists of distributed controllers acting in closed-loop on loads once the voltages at monitored transmission buses fall (and stay for some time) below threshold values. The upper level takes advantage of a WAMS to detect instability, and assign their voltage thresholds to the lower-level controllers, in the spirit of an adaptive system. The control scheme is robust with respect to controller or telecommunication failures.

The paper is organized as follows. To make the paper selfsupporting, some fundamentals of voltage instability and its countermeasures are recalled in Section 2. The lower and upper levels of the proposed scheme are detailed in Section 3 and 4, respectively. Section 5 is devoted to simulation results, while conclusions are provided in Section 6.

\section{Voltage instability mechanism and corrective controls}

This section reviews the basic voltage instability mechanism of a power system subject to a large disturbance [i]]. It uses a two-bus system example made as simple as possible while still capturing the main features of instability. Next, the two corrective controls considered in this work are briefly described.

\subsection{Voltage instability mechanism}

Consider the system in Fig. Z.a in which a generator feeds a load through a transmission line. It is assumed that the generator keeps its terminal voltage $V_{g}$ constant and provides any active power requested by the load. The line is simply represented by its series reactance $X$. Finally, the load is assumed to have unity power factor, i.e. it does not consume reactive power. The power flow equations of this system are:

$$
P=-\frac{V_{g} V}{X} \sin \theta \quad 0=-\frac{V^{2}}{X}+\frac{V_{g} V}{X} \cos \theta
$$

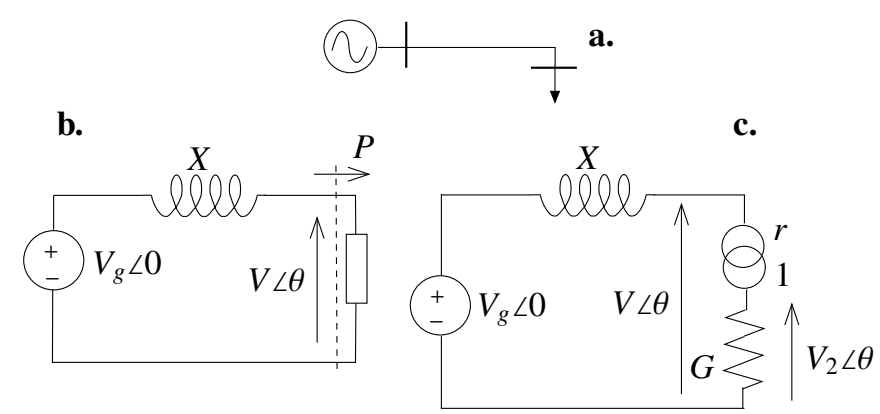

Figure 2: Two-bus system and corresponding circuits

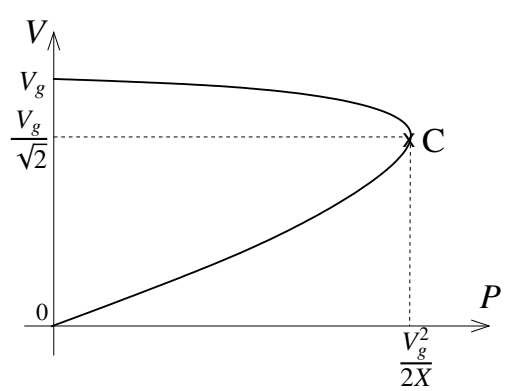

Figure 3: Network PV curve (load with unity power factor)

where all symbols are defined in Fig. 2.b. Combining these two equations, the voltage $V$ at the load bus is easily obtained as:

$$
V=\sqrt{\frac{V_{g}^{2}}{2} \pm \sqrt{\frac{V_{g}^{4}}{4}-X^{2} P^{2}}}
$$

The variation of $V$ with $P$ is shown in Fig. B where the upper (resp. lower) part of the curve corresponds to the solution with the $+($ resp. the - ) sign in (Z). Such a plot is referred to as "PV curve" by power system engineers. The load power is maximum at point $\mathrm{C}$, also called "critical" point. The maximum deliverable power is $P_{C}=\frac{V_{g}^{2}}{2 X}$ under voltage $V_{C}=\frac{V_{g}}{\sqrt{2}}$.

Load dynamics play an important role in voltage instability. In the long-term time frame, loads are controlled by Load Tap Changers (LTCs). These devices adjust the turn ratios of the transformers feeding distribution systems in order to keep the voltages at the distribution sides of the transformers close to a set-point. LTCs can act in discrete steps only, corresponding to the available transformer tap positions. They remain the main voltage control means in distribution systems, although this task is expected to be shared by distributed generation units in future smart grid architectures [8, 9].

To illustrate the LTC effect, in Fig. Z.c, the load of our twobus system has been replaced by a load at distribution level behind a step-down transformer. In line with the previous simplifications, this transformer is assumed ideal, thus:

$$
V_{2}=\frac{V}{r}
$$




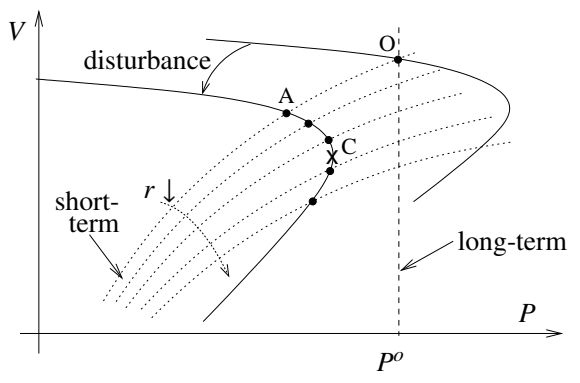

Figure 4: Voltage instability explained with (network and load) PV curves

where $r$ is the transformer ratio and $V_{2}$ the distribution voltage. Furthermore, a constant conductance load is considered:

$$
P=G V_{2}^{2}=G\left(\frac{V}{r}\right)^{2}
$$

Let $V_{2}^{o}$ be the voltage set-point of the LTC. Whenever $V_{2}$ falls below $V_{2}^{o}-\epsilon$ (resp. raises above $\left.V_{2}^{o}+\epsilon\right)$, the LTC decreases (resp. increases) $r$ in order to bring back $V_{2}$ in the dead-band $\left[V_{2}^{o}-\epsilon V_{2}^{o}+\epsilon\right] . r$ is changed step by step with delays between changes. The dead-band is needed for proper operation with a limited number of tap positions.

Network and load characteristics are combined in Fig. 田. Assume that the system operates initially at point $\mathrm{O}$. The dotted line passing through $\mathrm{O}$ is the transient load characteristic, corresponding to (四), while the vertical dashed line is the steadystate load characteristic. In steady state, assuming that the LTC has restored $V_{2}=V_{2}^{o}$ (neglecting the effect of $\epsilon$ ), the load power is $P=P^{o}=G\left(V_{2}^{o}\right)^{2}$. Thus, by controlling distribution voltages, LTCs make loads appear constant power in steady state.

The remaining of the figure illustrates a typical instability scenario. A severe disturbance, such as the outage of a transmission equipment, increases $X$ so much that the new maximum deliverable power (corresponding to point $\mathrm{C}$ ) falls below $P^{o}$, the power initially consumed by the load, that the LTC will try to restore indirectly. Since the long-term load characteristic does no longer intersect the network PV curve, it can be concluded that the system has lost its equilibrium. This will result in the following unstable evolution. Right after the disturbance, the new operating point is $\mathrm{A}$, at the intersection of the shortterm load and network post-disturbance characteristics. At this point, the load power is smaller than $P^{o}$, which means that $V_{2}$ is smaller than $V_{2}^{o}$. Hence, the LTC will decrease $r$ in successive steps. As can be seen from (田), each new value of $r$ yields a new short-term load characteristic. The intersections of those characteristics with the network PV curve give the successive operating points shown with dots in Fig. 目: in its unsuccessful attempt to restore the distribution voltage, the LTC depresses the transmission voltage $V$. After crossing the maximum power point $\mathrm{C}$, the tap changes are even counterproductive!

A similar behaviour is observed in Fig. 四 where the distribution voltage (dashed line) cannot be restored in the corresponding [0.99 1.01] pu dead-band.

Expectedly, some features of voltage instability are not shown in the above simplistic example [I, [10]:
- an excessive decline of voltages is unacceptable since it may cause further equipment (in particular loads) to trip or it may trigger a faster instability such as loss of synchronism or motor stalling (see the final evolution in Fig. Ш);

- generators are not pure voltage sources. In normal conditions, the terminal voltage of a synchronous generator is tightly controlled by its automatic voltage regulator. However, if keeping that voltage constant requires producing too much reactive power, the generator field current may exceed its thermal limit and be reduced after some delay by an Over-Excitation Limiter (OEL) to avoid equipment damages. The resulting loss of voltage control by a generator has a similar effect as the initiating outage: the PV curve further shrinks, and the maximum power deliverable to the load further decreases;

- besides LTCs, there are other load power restoration mechanisms such as the control of heating loads by thermostats.

\subsection{Corrective controls}

The above simple example suggests directly two types of corrective controls, which are considered in this paper:

1. modified LTC control: to stop the degradation caused by LTCs. Standard emergency actions consist of blocking tap changers on their current positions, moving them to predetermined positions, or reducing the voltage set-points $V_{2}^{o}$ [10-112]. In this paper a more advanced control is considered which involves a transmission voltage preserving logic [113];

2. load shedding: moves the long-term load characteristic to the left, in Fig. 田, so that a new intersection with the network PV curve is created. Curtailing a proper amount of interruptible load at the proper place and in appropriate time is a very effective countermeasure against voltage instability [10, [14-18]. It is needed when system voltages drop too much immediately after the disturbance, the LTCs being to slow to move. At the price of disconnecting some loads, the others quickly regain normal voltage. Shedding is actuated by opening distribution circuit breakers; finer control of individual appliances is envisioned in the context of demand response management in smart grids [ $[8,9]$.

The above corrective controls affect the quality of power supply, and, hence, are envisaged in severe situations only. Less intrusive controls, to be actuated in priority, consist of increasing generator voltages (though in a limited interval) or connecting shunt capacitors (if available) to the transmission bus feeding the load. Both actions increase to some extent the maximum power deliverable to the load. A more detailed discussion of emergency voltage stability controls can be found in [19, 20].

\section{Lower-level distributed control of load shedding and tap changers}

\subsection{Distributed control}

The design of an emergency control scheme against voltage instability must address a number of issues: 
- the magnitude of the corrective action needed to stabilize the system is not known beforehand;

- saving the system from a blackout is the priority. To this end, the corrective action can be slightly in excess, in exchange for other advantages such as reliability. Nevertheless, since corrective control is intrusive from the consumer viewpoint, it should be as small as possible;

- real-life power systems include a large number of loads and generators. The regions exposed to voltage instability are known by transmission system operators. However, inside those regions, it is not known beforehand where it is most appropriate to act. This is even more true for well meshed networks;

- loads are the most effective components to act on. However, in practice, there is much uncertainty regarding their behaviour. For instance, when opening a distribution circuit breaker, the controller does not necessarily know how much reactive power is going to be curtailed together with the active power, and how much the power of the remaining loads will vary with the voltage increase;

- the scheme must be robust with respect to failures of sensors or actuators. This requires some form of redundancy.

Most of the above issues are addressed by resorting to a set of controllers distributed over the region of interest, and operating in closed-loop. "Closed-loop operation" refers to the ability for a controller to act in successive steps, each decision relying on the measured result of previous actions. The distributed design allows adjusting corrective control to the disturbance location [21]. It also provides redundancy, since in case of failure of one controller, the others can take over [22].

Two additional features of the distributed controllers contribute to making the scheme simple and, hence, more reliable:

- the controllers do not exchange information, but are rather informed of their respective actions through the power system itself. This is made possible by the absence of "inertia" in voltage dynamics: in the time frame of concern, it can be considered that the effects of one controller are instantaneously felt by the others;

- the controllers do not need any system model.

In the sequel a combination of distributed load shedding and LTC controllers is considered, as sketched in Fig. 5. Each load shedding controller monitors the voltage $V$ at a transmission bus and acts on a set of loads at distribution level, located close enough to the monitored bus, so that their curtailment allows increasing $V$. Each LTC controller measures the voltage of both the transmission and the distribution terminal of the transformer it is controlling. In normal operating conditions, it regulates the distribution voltage as usual, while in emergency conditions, it preserves the transmission voltage. The remaining of this section is devoted to presenting these controllers in greater detail.

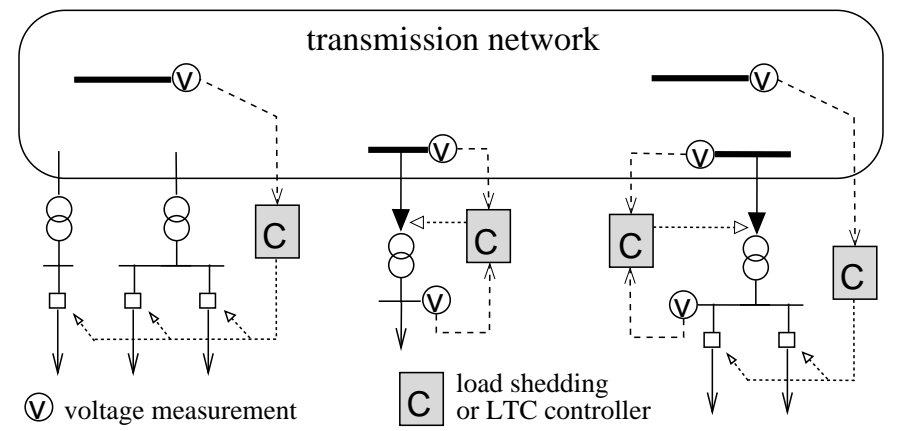

Figure 5: Distributed control of load shedding and tap changers

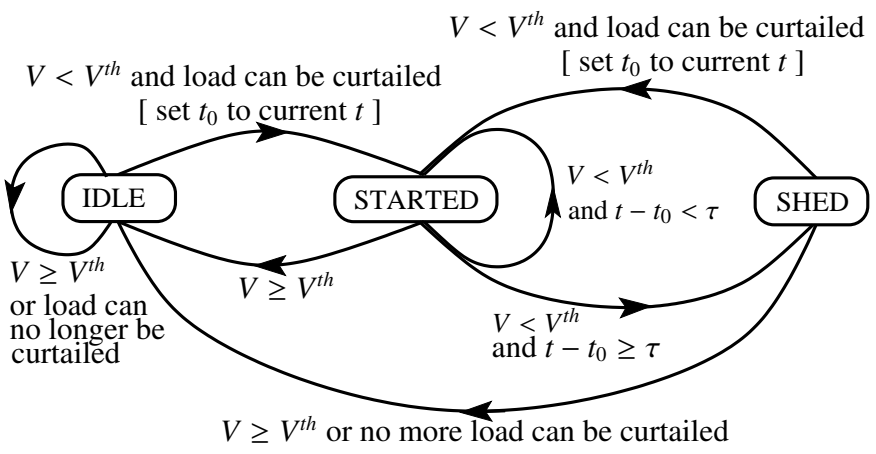

Figure 6: Logic of individual load shedding controller. Within brackets: action taken when the transition takes place [22]

\subsection{Load shedding controller}

Each load shedding controller relies on the simple rule:

$$
\text { IF } V<V^{\text {th }} \text { during time } \tau \text {, shed load power } \Delta P
$$

A more precise description is given by Fig. 6 in the form of an automaton. As long as $V$ remains above $V^{\text {th }}$, the controller is idle, while it is started as soon as a disturbance causes $V$ to drop below $V^{t h}$. Let $t_{o}$ be the time where this change takes place. The controller remains started until either the voltage recovers, or the time $\tau$ is elapsed since $t_{o}$. In the latter case, the controller sheds a power $\Delta P$ and returns to either idle (if $V$ recovers above $V^{\text {th }}$ or no more load can be shed) or started state (if $V$ remains below $V^{\text {th }}$ and there remains load to shed). In the second case, the current time is taken as the new value of $t_{o}$ and the controller is ready to act again.

The choice of $V^{\text {th }}$ will be discussed in detail in Section 4 . As regards $\tau$ and $\Delta P$, they can be adjusted to the severity of the situation, assessed through the amplitude of $V^{\text {th }}-V$ [22]. In fact, our analysis of many cases has shown that small $\tau$ and $\Delta P$ values, i.e. shedding with little delay and in small steps, usually lead to shedding less load. It also makes the system recover more smoothly. Thus, constant, pre-defined values of $\tau$ and $\Delta P$ are considered in this paper. Note that $\tau$ must be large enough so that the controller does not to react to a short-circuit on the transmission system. Indeed, after the fault has been cleared by protections, the voltage takes one or two seconds to recover a normal value. 


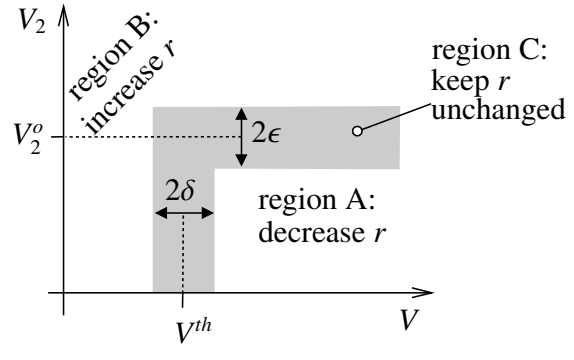

Figure 7: LTC control combining DVR and TVP logics

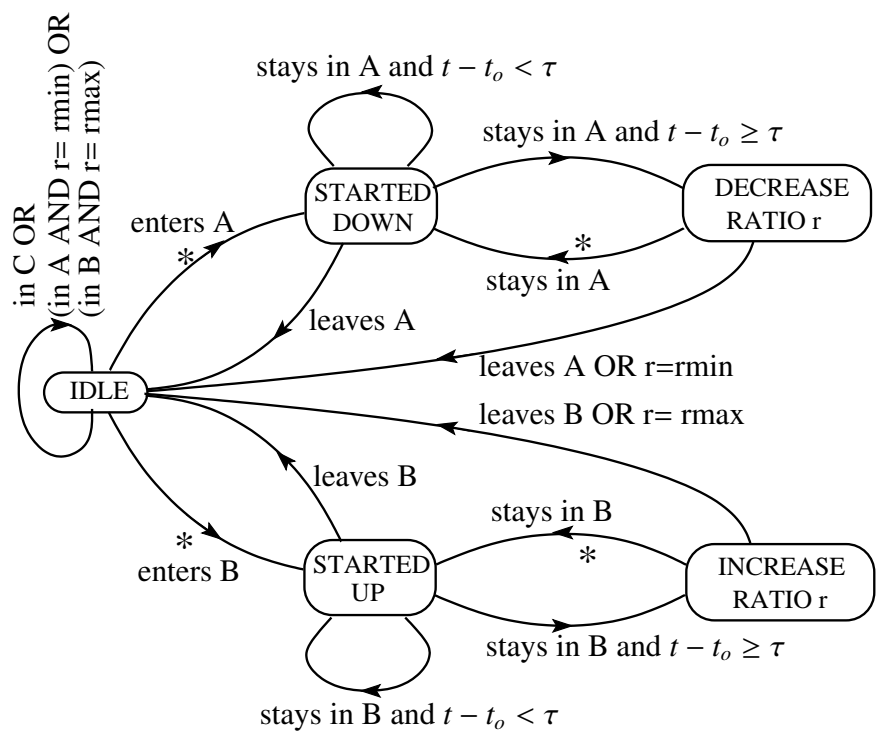

Figure 8: Combined DVR-TVP logic of individual LTC controller. A, B and C refer to the regions of operation in Fig. Z. A star (*) indicates that $t_{o}$ is set to current time $t$ when the transition takes place. "rmin" (resp. "rmax") denotes the minimum (resp. maximum) value of the ratio. For clarity, adjustments of $\tau$ (different delays before first and between subsequent tap changes) are not shown.

\subsection{Load Tap changer controller}

With the transformer ratio defined as in Fig. \.c, a standard LTC controller monitors the distribution voltage $V_{2}$ and modifies the ratio $r$ as follows: decrease $r$ if $V_{2}<V_{2}^{o}-\epsilon$, increase $r$ if $V_{2}>V_{2}^{o}+\epsilon$, and leave it unchanged otherwise. A more advanced control logic is considered here, in which the transmission voltage $V$ (on the high-voltage side of the transformer) is also monitored with respect to a threshold value $V^{\text {th }}$. As long as $V$ remains above $V^{t h}+\delta$ the LTC operates in the above, standard manner. On the contrary, if $V$ falls below $V^{\text {th }}-\delta$, the LTC stops regulating the distribution voltage and, instead, preserves the transmission voltage by increasing $r$. If $V$ lies in the $\left[V^{t h}-\delta V^{t h}+\delta\right]$ range, the tap changer does not move; this deadband prevents from oscillating between the Distribution Voltage Regulation (DVR) and the Transmission Voltage Preservation (TVP) logics. The above rules are summarized in Fig. $\square$ while the combined DVR-TVP logic is presented in automaton form in Fig. 8.

The choice of $V^{t h}$ is discussed in detail in Section 田. The dead-band $2 \epsilon$ is unchanged with respect to usual LTCs, with a typical value in the range of $2 \%$. The dead-band $2 \delta$ can be given the same value.

\subsection{Automatic shunt compensation switching}

As already mentioned, this paper focuses on LTC control and load shedding. However, the switching of shunt compensation (switching on capacitors - switching off reactors) is handled similarly, and can be described in similar terms. Usually, several capacitor banks are available in a substation. Once the transmission voltage $V$ falls below $V^{\text {th }}$, the local controller switches one bank at a time, after a delay similar to $\tau$ in Fig. 6 .

\section{Upper level: emergency detection and voltage threshold adjustment}

\subsection{Motivation for an upper level}

In spite of their advantages, the distributed controllers still rely on the particular value assigned to $V^{t h}$. This voltage threshold should not be set too high, to avoid triggering corrective control at normal voltages, or at low but stable voltages. It should not be set too low either, since it will lead to act later and, hence, expose the system to degraded operating conditions for a longer time. Also, when delayed, the corrective actions have to be more pronounced [I]], which results in sharper voltage corrections. Finally, Section 5.5 will give an example where it is impractical to rely on $V^{\text {th }}$ without further information.

This leads to complementing the distributed controllers with an upper level in charge of sending $V^{\text {th }}$ values - or equivalent information - in real-time, when emergency conditions and impending instability are detected.

The aim of an upper-level controller in a hierarchical control scheme is typically to coordinate the efforts of lower-level controllers or to adjust their parameters adaptively. The latter option is considered in this work. By adjusting $V^{\text {th }}$ in response to the particular disturbance faced, the upper level provides the whole scheme with the basic feature of an adaptive system.

To this purpose, the upper level is assumed to embed a WAMS receiving real-time measurements [7]. In the future, WAMS are expected to benefit from the progress made in synchronized phasor measurement technology [6].

\subsection{Implementation}

Following a large disturbance, the role of the upper level is to detect that the system is evolving towards long-term voltage instability, more precisely to identify that the system has crossed a "critical voltage profile". The latter defines the lowest acceptable voltage $V^{\text {th }}$ at the various buses monitored by the lower-level controllers. This way of doing is justified by the observation that a long-term voltage unstable system undergoes a monotonic decrease of voltages, as illustrated by Fig. $\square$. Then, the role of the distributed controllers will be to restore and maintain their monitored transmission voltages at or slightly above the critical voltage profile. 
This can be performed with very little information sent from upper to lower level (virtually a single bit of information!). Indeed, at the time the upper level detects that the system has crossed the critical voltage profile, it sends a signal to the lowerlevel controllers requesting each of them to take its currently measured voltage $V$ as threshold value $V^{\text {th }}$. Each controller acts independently from the others to restore $V \geq V^{t h}$.

If, for any reason, the upper level monitoring scheme fails providing the signal to the local controllers, the latter may use their original (preset) thresholds. This redundancy increases the reliability of the whole protection.

\subsection{Detecting an impending instability}

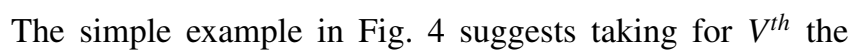
voltage $V_{C}$ at the critical point $C$. Indeed, at this point, the load power is restored to the greatest possible extent. Before crossing $\mathrm{C}$, the transmission voltage $V$ is depressed by the tap changes but in exchange for a restoration of the distribution voltage $V_{2}$, while after crossing $C$ both transmission and distribution voltages are depressed.

The method detailed in [23] can be used to identify the critical point in the general case involving multiple loads and multiple generators, some of them getting limited. It is summarized hereafter.

A set of algebraic equations:

$$
\phi(z, s)=\mathbf{0}
$$

is fit to the sampled states, where $z$ denotes the state vector and $\boldsymbol{s}$ the vector of load active and reactive powers. These equations are obtained under the following assumptions:

- the network is represented by its bus admittance matrix, using real-time breaker status information;

- the short-term dynamics of generators, automatic voltage regulators, speed governors, static var compensators, etc. are replaced by accurate equilibrium equations;

- whether a generator is voltage controlled or limited by its OEL is either known from measurements or detected from computation of the field current.

Sensitivities identify when a combination of load active and reactive powers passes through a maximum. This requires knowing the consumed powers only: no model of load behaviour with voltage is needed. Sensitivities of the total reactive power generation $Q_{g}$ to the load reactive powers are considered. They are obtained by differentiation of (6) as [20]:

$$
\frac{\partial Q_{g}}{\partial \boldsymbol{q}}=-\left(\frac{\partial \phi}{\partial \boldsymbol{q}}\right)^{T}\left(\left(\frac{\partial \phi}{\partial z}\right)^{T}\right)^{-1} \frac{\partial Q_{g}}{\partial z}
$$

where $\boldsymbol{q}$ is the vector of load reactive powers, which is a subvector of $s$.

At the maximum load power point, one real eigenvalue of $\frac{\partial \phi}{\partial z}$ passing through infinity. In practice, the critical point crossing is identified at a discrete time $k$ such that, for at least bus $j$ :

$$
\frac{\partial Q_{g}}{\partial q_{j}}(k-1)>d \text { and } \frac{\partial Q_{g}}{\partial q_{j}}(k)<-d
$$

where $d>0$ is a properly chosen threshold.

It is essential to reflect any change of generator voltage control in the equations (6). An estimate of $E_{q}$, the e.m.f. proportional to field current, is used to identify whether a synchronous generator operates under control of its Automatic Voltage Regulator (AVR) or has been already limited by its OEL. Under AVR control, an equation such as:

$$
k E_{q}^{s}-G\left(V^{o}-V\right)=0
$$

is used, while under OEL control, it is replaced by an equation of the type:

$$
E_{q}-E_{q}^{l i m}=k E_{q}^{s}-E_{q}^{l i m}=0
$$

where $G$ is the open-loop static gain of the AVR, $E_{q}^{s}$ is the e.m.f. behind saturated synchronous reactances, $k$ is the saturation factor, $V$ is the terminal voltage, $V^{o}$ is the AVR voltage set-point, and $E_{q}^{\lim }$ corresponds to the field current forced by the OEL. $E_{q}$ and $k$ are components of $z$ together with bus voltages and other variables.

The most important feature of the sensitivities is their ability to anticipate the effect of an approaching OEL activation. To this purpose, when $E_{q}$ exceeds $E_{q}^{\lim }$, the OEL equation (10) is anticipatively substituted to the AVR equation (Q) before evaluating $\frac{\partial \phi}{\partial z}$. More details can be found in [23]. The anticipation capability is illustrated in the next section.

\subsection{Real-time measurement requirements}

The above sensitivities are intended to be computed on successive "snapshots" of the evolving system state. For each snapshot, the complex voltages at the various buses should be estimated (at least in the region of interest). This is typically the role of a state estimator running in the control center. In the authors' simulations, the rate at which measurement samples are processed is high compared to the capability of present day static state estimators. Therefore, extensions towards tracking state estimation have to be considered. In this respect, the emergence of the time synchronized phasor measurement technology opens new perspectives, as detailed in [24], for instance.

Further discussion of this important issue is beyond the scope of this paper. Note, however, that sensitivity computation and monitoring can be replaced by a simpler (more conservative) detection of emergency voltage conditions. In the two-level scheme proposed in this paper, what is eventually sought is an emergency signal sent to the lower-level controllers.

\section{Simulation results}

\subsection{Test system}

The performance of the proposed controller is illustrated on the 74-bus, 20-generator, 22-load Nordic32 system. Its one-line 


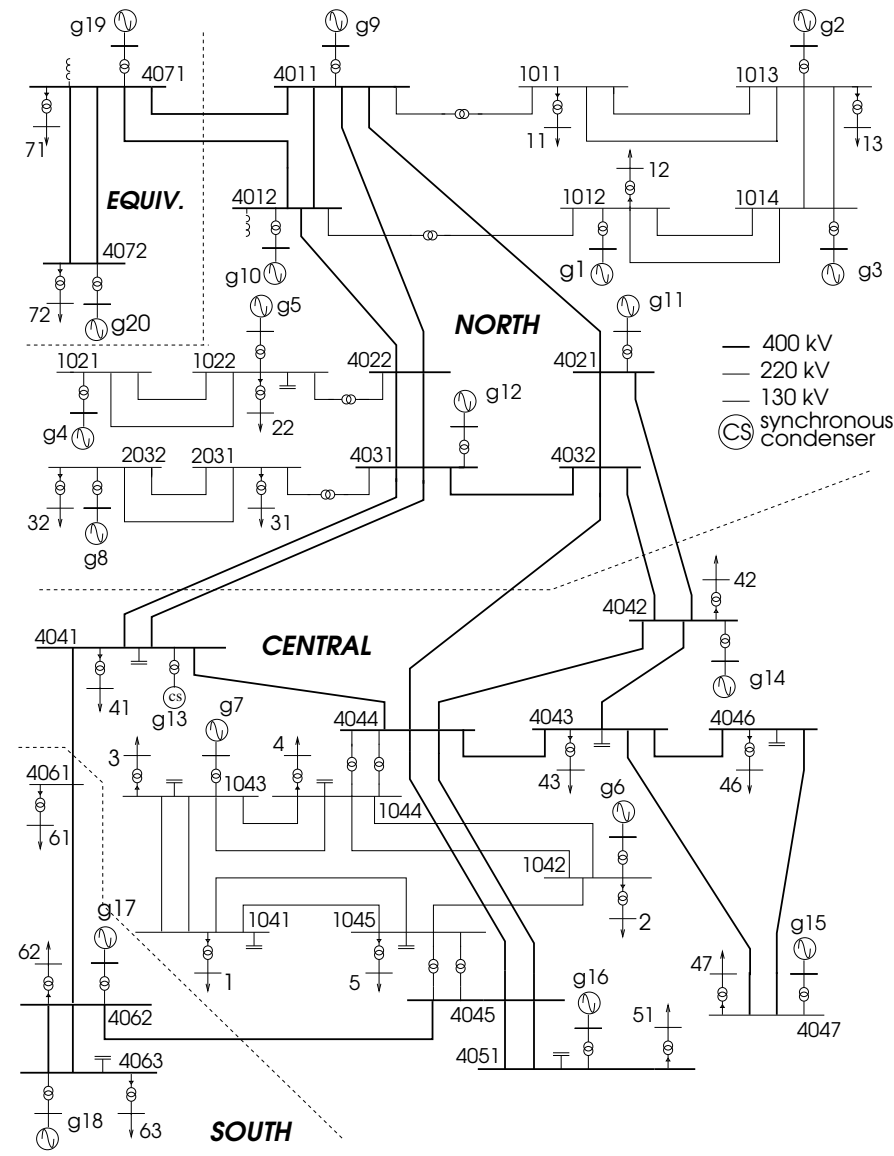

Figure 9: One-line diagram of the Nordic32 test system

diagram is shown in Fig. $Q$. This small but realistic test system has been used in several references, for instance [22, 23]

The results were obtained from time simulations of the system model under the phasor approximation, typical of power system stability studies [25]. This model involves close to 700 differential and algebraic states (including the 148 real and imaginary components of the 74 complex bus voltages) Each generator is equipped with an OEL in charge of limiting its field current after a delay that may depend on the thermal overload. Each of the 22 loads is fed through a distribution transformer equipped with LTC. The LTCs have various delays: on the average, $30 \mathrm{~s}$ on the first tap change, and $10 \mathrm{~s}$ between subsequent changes. There are around 250 discrete states in limiters, switches, LTC discrete-time controllers, etc.

\subsection{Disturbance}

The disturbance of concern is a short-circuit at $t=1 \mathrm{~s}$ on line 4032-4044. This fault is cleared after $0.1 \mathrm{~s}(5$ cycles at $50 \mathrm{~Hz})$ by opening the faulted line, which remains opened. As the initial operating state is insecure, the outage of that line makes the system long-term voltage unstable.

\subsection{Lower-level distributed controllers}

To counteract voltage instability, the system has been provided with five load shedding controllers, distributed over the
$130-\mathrm{kV}$ network of the Central area, where the most pronounced voltage drops are experienced. These controllers monitor voltages at buses 1041, 1042, 1043, 1044, and 1045, respectively, and shed load at the corresponding distribution buses 1 , 2, 3, 4 and 5 (see Fig. Q). Each controller sheds load in steps $\Delta P$ of $10 \mathrm{MW}$ (with a corresponding decrease of the reactive power) and with a delay $\tau$ of 3 seconds.

In replacement of, or in complement to load shedding, the combined DVR-TVP logic detailed in Fig. $\mathbb{Q}$ has been considered for the LTCs controlling the same five buses. $\delta$ has been set to $0.01 \mathrm{pu}$, which is also the value of $\epsilon$ (see Fig. \).

\subsection{Results with exponential load models}

The results of this subsection have been obtained assuming that all loads follow the so-called exponential model [25]:

$$
P=P^{o}\left(\frac{V}{V^{o}}\right)^{\alpha} \quad Q=Q^{o}\left(\frac{V}{V^{o}}\right)^{\beta}
$$

where $V$ is the corresponding distribution bus voltage, $V^{o}$ the initial value of $V, P^{o}$ (resp. $Q^{o}$ ) the initial load active (resp. reactive) power. $\alpha=1, \beta=2$ was chosen for all loads.

\section{Uncontrolled system response}

The long-term unstable evolution of the uncontrolled system has been shown in Fig. 1 . It is driven by the LTCs and by a cascade of OEL activations taking place between $t=55$ and $t=147 \mathrm{~s}$, on g12, g14, g7, g11, g6, g15 and g16, successively.

\section{Instability detection at upper level}

At the upper level, the sensitivities (ㅁ) are used as explained in Section 4. The $z$ vectors have been obtained from snapshots of the bus voltage phasors provided by time simulation.

The evolution of the sensitivity $\frac{\partial Q_{g}}{\partial q_{j}}$ relative to bus 1041 is shown in Fig. 100. A single bus is considered for clarity, but it is proved in [23] that the sensitivities change sign altogether (at least at buses most impacted by instability).

The solid line correspond to the sensitivity evaluated "without OEL anticipation", i.e. accounting for the field current limitation of a generator after its OEL has acted. The change in sign takes place at $t=87 \mathrm{~s}$. Assuming a one-second delay to ascertain that the sensitivities settle to negative values, the emergency signal is sent by the upper level at $t=88 \mathrm{~s}$. The dashed line shows the sensitivity computed "with OEL anticipation", i.e. accounting for the near-future limitation of a generator as soon as the field current exceeds its limit. The change in sign occurs at $t=72 \mathrm{~s}$, i.e. $15 \mathrm{~s}$ earlier.

When receiving the emergency signal, each lower-level controller takes the transmission voltage it currently measures as threshold value $V^{\text {th }}$. Since the sensitivities give an early warning, some of these voltages can still be high. Considering that emergency control is intrusive for customers, $V^{\text {th }}$ has been upper limited to $0.95 \mathrm{pu}$. The resulting values are given in Table $\mathbf{D}$. 
$\square$

$\square$ 
Figure 12: Evolution of load powers (shedding actions are identified with *)

Table 3: Power curtailments (in MW) with inter-level communication failures

\begin{tabular}{ccccccc}
\hline & \multicolumn{2}{c}{ No failure } & \multicolumn{2}{c}{ Case A } & \multicolumn{2}{c}{ Case B } \\
bus & $V^{\text {th }}$ & power & $V^{\text {th }}$ & power & $V^{\text {th }}$ & power \\
& $(\mathrm{pu})$ & shed & $(\mathrm{pu})$ & shed & $(\mathrm{pu})$ & shed \\
\hline 1041 & 0.93 & 20 & 0.93 & 40 & 0.93 & 80 \\
1042 & 0.95 & 0 & 0.95 & 0 & 0.95 & 0 \\
1043 & 0.95 & 50 & 0.95 & 50 & 0.95 & 130 \\
1044 & 0.92 & 30 & 0.92 & 100 & 0.90 & 0 \\
1045 & 0.94 & 100 & 0.90 & 0 & 0.90 & 0 \\
\hline Total & & 200 & & 190 & & 210 \\
\hline
\end{tabular}

The LTC operation is illustrated in Fig. 14, relative to transformer 1041-1. The figure shows the trajectory of the system superimposed to the diagram of Fig. 7. The large transients due to the initial short-circuit have been removed for clarity. The pre-disturbance operating point is $\mathrm{O}$, with the voltage at the distribution bus 1 in its dead-band. At $t \simeq 30 \mathrm{~s}$, when the system has almost settled to a short-term equilibrium (see Fig. 1), but before the LTC starts acting, the operating point is A. The unsuccessful operation of the LTC leads to point B. Then, the situation is aggravated by the field current limitation of generators g14 and g7, leading to point C. This makes the LTC enter the TVP region of operation. The reverse tap changes preserve transmission to the detriment of distribution voltage, until the LTC eventually settles at point D in the dead-band.

\section{Comparison}

When resorting to load shedding, some loads are disconnected while the others regain a normal voltage value. With LTC control, the effort is shared by all loads, whose powers decrease under the effect of depressed voltages. These two actions on loads can be compared considering, for load shedding, the total curtailed power and, for LTC control, the non-restored load power [13]:

$$
\Delta P_{n r}=\sum_{i \in \mathcal{I}}\left(P_{i}^{o}-P_{i}^{f i n a l}\right)
$$

Figure 13: Evolution of voltage at bus 1041 with DVR-TVP control of LTCs (two variants)

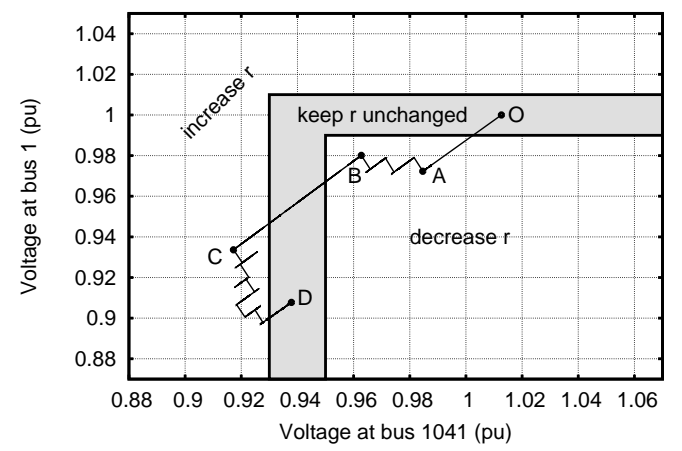

Figure 14: System trajectory in the $\left(V_{1041}, V_{1}\right)$ space (voltages at the transmission and distribution ends of transformer 1041-1)

where $P_{i}^{o}$ is the pre-disturbance active power of the load at the $i$-th bus, $P_{i}^{\text {final }}$ is the corresponding value in the final steady state, and $\mathcal{I}$ is the set of distribution buses whose voltage is outside the LTC dead-band.

The values of $\Delta P_{n r}$ and the contribution of each bus are given in Table 4 for the two stabilized evolutions shown in Fig. ए3]. The values of $\Delta P_{n r}$ are comparable to the total curtailed powers detailed in Table Q.

\subsection{Results in the presence of induction motor loads}

The performance of the proposed scheme has been tested in more stringent conditions, assuming that the loads at buses 1 , $2,3,4,5,43,46$, and 47 include $40 \%$ of induction motors. To this purpose each of these eight loads has been split into a part represented with the previous exponential model, taking $60 \%$ of the initial active power, in parallel with an equivalent (single cage) induction motor, as shown in Fig. 15, relative to bus 1 . Each equivalent motor accounts for many motors connected to the distribution system. It is represented by a set of three differential and two algebraic equations, with parameters typical of large industrial motors [10]. Shedding is applied to the nonmotor part of the loads. 


\begin{tabular}{ccc}
\multicolumn{3}{c}{ Table 4: Non-restored load power (MW) } \\
\hline bus & $\begin{array}{c}\text { without OEL } \\
\text { anticipation }\end{array}$ & $\begin{array}{c}\text { with OEL } \\
\text { anticipation }\end{array}$ \\
\hline 1 & 55 & 56 \\
2 & 0 & 0 \\
3 & 26 & 17 \\
4 & 73 & 108 \\
5 & 64 & 39 \\
\hline Total & 218 & 220
\end{tabular}

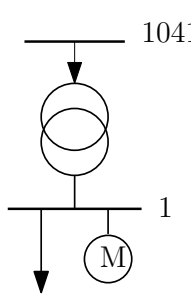

Figure 15: Composite load model including an equivalent motor

\section{Uncontrolled system response}

The evolution of the voltage at bus 1041, under the effect of the same disturbance, is shown with solid line in Fig. ए6. For comparison purposes, the figure shows with dotted line the response which was obtained with fully exponential load model, see Fig. $\square$. In the presence of motors, the voltage drop is sharper. It takes place earlier, when the voltage support of key generators is lost due to OEL action, which causes the motors to stall [1, [10]. The task of designing emergency control is more delicate because: (i) a few seconds before collapsing, voltages still have normal values, and (ii) the decrease is so fast that prompt corrective actions are needed.

\section{Instability detection at upper level}

The evolution of the component of $S_{Q_{g} q}$ relative to bus 1041 is shown in Fig. [17. Without (resp. with) OEL anticipation, the change in sign takes place at $t=68 \mathrm{~s}$ (resp. $t=53 \mathrm{~s}$ ).

The values of $V^{\text {th }}$ taken by the controllers when receiving the upper-level emergency signal are given in Table [5. With OEL anticipation, the emergency was detected so early that all voltages were still above the upper bound of $0.95 \mathrm{pu}$.

\section{Stabilization by DVR-TVP control of LTCS}

The effect of LTCs operating according to the combined DVR-TVP logic is shown in Fig. 18. Even though the signal is received very early, the LTCs control actions are too slow to prevent the sharp voltage decay. They only succeed postponing voltage collapse a little, at the cost of pronounced oscillations.

\section{Stabilization by load shedding}

Failure to stabilize the system also takes place when resorting to distributed load shedding controllers with $V^{\text {th }}=0.90 \mathrm{pu}$, as previously considered (see Table $\square$ ). Indeed, with $\tau$ set to three seconds, the controllers react too late to stabilize the system. Reducing $\tau$ is not an option due to the risk of unduly shedding load after a short-circuit normally cleared by protections [26].

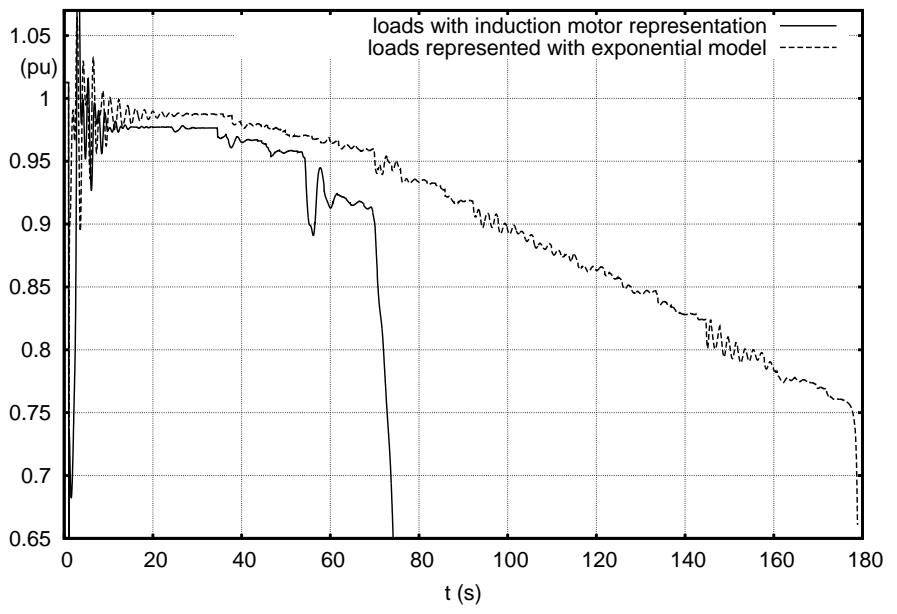

Figure 16: Unstable evolution of voltage at bus 1041 in the presence of induction motor loads and with exponential load models

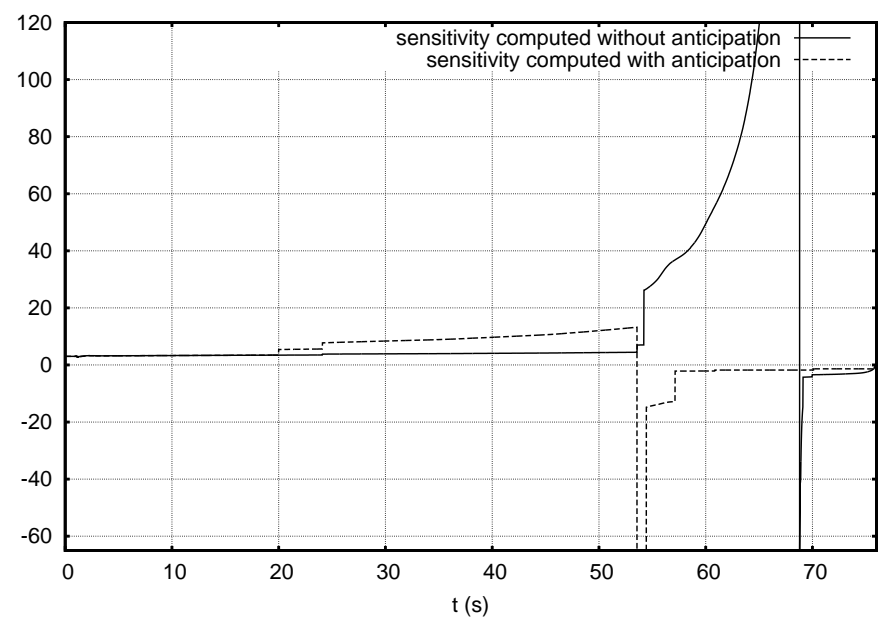

Figure 17: Evolution of the sensitivity $\frac{\partial Q_{g}}{\partial q_{j}}$ relative to bus 1041

Successful stabilization is obtained by combining load shedding with the upper-level early detection of emergency. The stabilized voltage evolution is shown in Fig. 119, for both sets of $V^{t h}$ values given in Table [5. The smoother evolution obtained with the higher $V^{\text {th }}$ settings is noteworthy.

Figure 20 shows the performance of the same load shedding scheme for 30,40 and $50 \%$ of motor load, respectively. Table 6 shows the corresponding power curtailments. The protection scheme performs very similarly in all three cases, which reveals some robustness with respect to load behaviour uncertainty.

\section{Stabilization by both LTC control and load shedding}

To deal with such severe situations, it is reasonable to combine both types of corrective control. This is illustrated in Fig. 2]. The curve with solid line shows the voltage evolution that result from DVR-TVP control of LTCs, based on the $V^{\text {th }}$ values in the third column of Table [1, together with load shedding, based on the $V^{\text {th }}$ values in the second column of the same table. Thus, LTC control is activated first, followed by 


\begin{tabular}{ccc}
\multicolumn{3}{c}{ Table 5: Voltage thresholds $V^{\text {th }}$ (in pu) } \\
\hline bus & $\begin{array}{c}\text { without OEL } \\
\text { anticipation }\end{array}$ & $\begin{array}{c}\text { with OEL } \\
\text { anticipation }\end{array}$ \\
\hline 1041 & 0.91 & 0.95 \\
1042 & 0.95 & 0.95 \\
1043 & 0.93 & 0.95 \\
1044 & 0.91 & 0.95 \\
1045 & 0.93 & 0.95
\end{tabular}

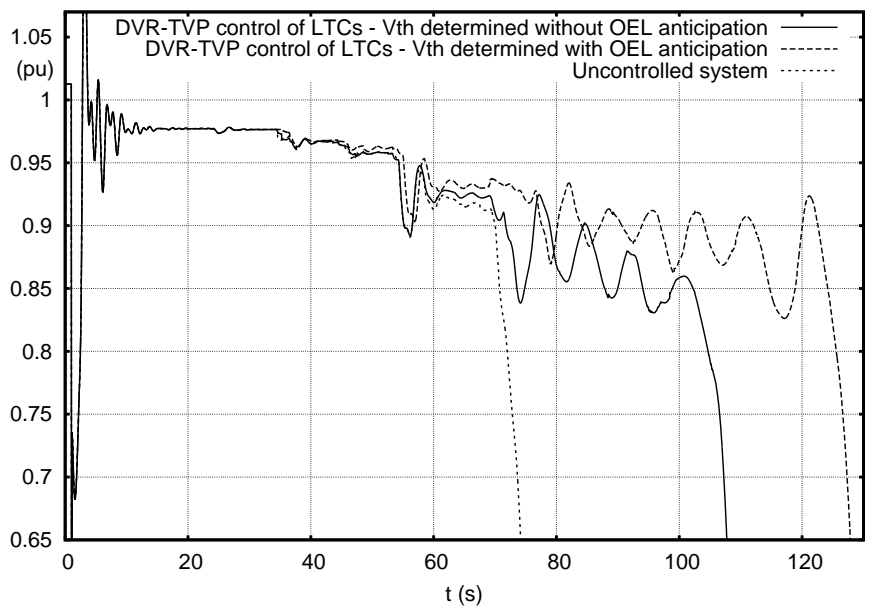

Figure 18: Unsuccessful system stabilization by DVR-TVP control of LTCs

load shedding. The dashed curve, reproduced from Fig. एप9, corresponds to load shedding only. The reduction of distribution voltages by LTCs leads to shedding only $60 \mathrm{MW}$, instead of $220 \mathrm{MW}$ when LTCs are not used for emergency control. In this combined scheme, two signals would be sent by the upper level, intended to LTCs and load shedding, respectively.

\section{Conclusion}

A two-level adaptive control scheme against power system voltage instability has been proposed.

At the lower level, it relies on a set of distributed controllers, acting when (and as long as) monitored transmission voltages are below threshold values. Upon detection of an emergency situation, the upper level sends a signal to the distributed controllers, for the latter to set their threshold values at the currently measured voltages. The objective is to keep system voltages above the critical profile corresponding to the emergency detection. The distributed controllers adjust their actions to the severity and location of the disturbance.

The emergency signal is obtained from wide-area monitoring by the upper level. To this purpose, an advanced sensitivitybased analysis has been considered.

The results obtained with the small but realistic Nordic 32 test system have confirmed the following expectations:

- proper adaptation of the voltage thresholds in real time yields a more dependable protection;

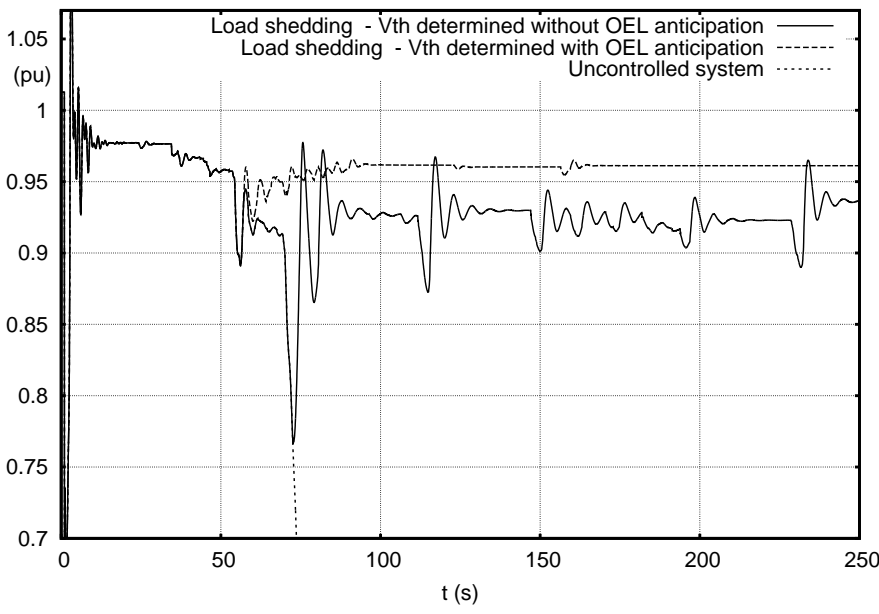

Figure 19: Evolution of voltage at bus 1041 with load shedding (two variants)

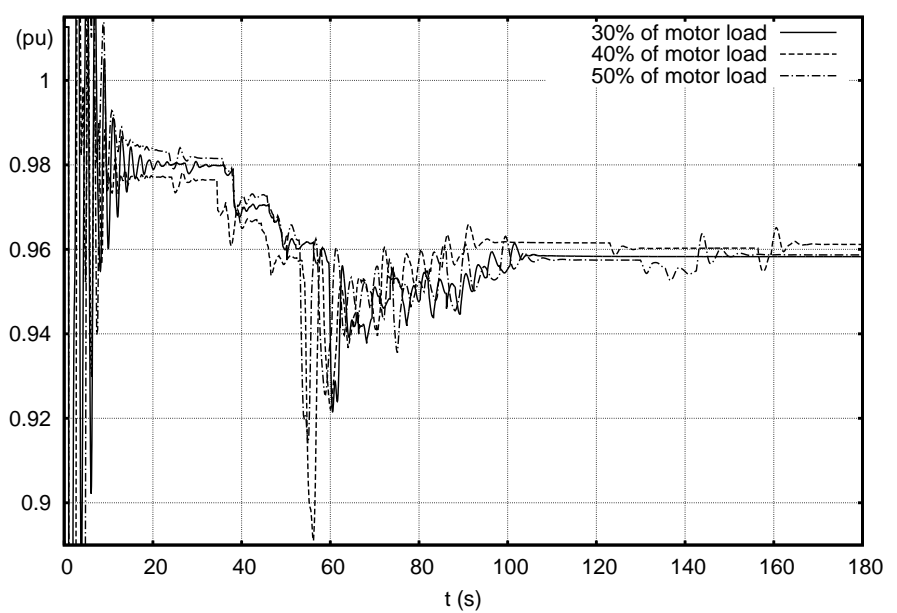

Figure 20: Evolution of voltage at bus 1041 with load shedding, for three proportions of motor load

- the proposed scheme is fault-tolerant since it accommodates failures to receive the emergency signal;

- the early emergency signal sent by the upper level allows acting fast enough in case of sharp voltage drops caused by motor loads, a situation where relying on local voltages is not sufficient.

Two types of emergency controls were considered. While modified control of load tap changers can help in slowly decreasing voltage situations, it has to be replaced or, even better, complemented by load shedding to counteract the faster instability caused by induction motor loads.

Automatic shunt compensation switching can be also included, and should be given priority as it is less intrusive for the consumers.

\section{Acknowledgment}

B. Otomega has been financially supported by the Sectoral Operational Programme Human Resources Development 2007- 
Table 6: Power curtailments (MW)

\begin{tabular}{cccc}
\multicolumn{4}{c}{ Table 6: Power curtailments (MW) } \\
\hline bus & $30 \%$ & $40 \%$ & $50 \%$ \\
\hline 1041 & 30 & 30 & 20 \\
1042 & 0 & 0 & 0 \\
1043 & 0 & 0 & 10 \\
1044 & 130 & 120 & 130 \\
1045 & 0 & 0 & 0 \\
\hline Total & 160 & 150 & 160 \\
\hline
\end{tabular}

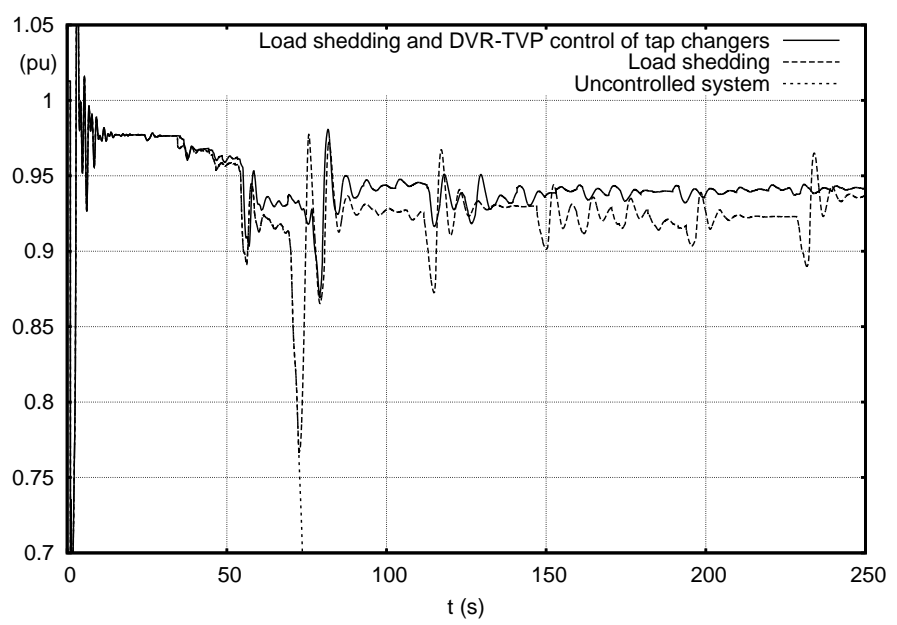

Figure 21: Evolution of voltage at bus 1041 with DVR-TVP control of LTCs and load shedding

2013 of Romanian Ministry of Labor, Family and Social Protection through Agreement POSDRU/89/1.5/S/62557.

\section{References}

[1] T. Van Cutsem, C. Vournas, Voltage Stability of Electric Power Systems, Springer (previously Kluwer Academic Publishers), Boston, 1998.

[2] P. Kundur, J. Paserba, V. Ajjarapu, G. Andersson, A. Bose, C. Cañizares, N. Hatzyargyriou, D. Hill, A. Stankovic, C. Taylor, T. Van Cutsem, V. Vittal, Definition and classification of power system stability, IEEE Trans. Power Syst. 19 (2004) 1387-1401.

[3] US-Canada Power System Outage Task Force, Final report on the August 14, 2003 blackout in the United States and Canada: Causes and recommendations, [Online]. Available: reports.energy.gov.

[4] Union for the Co-ordination of Transmission of Electricity, Final report: System disturbance on 4 November 2006, [Online]. Available: www.entsoe.eu.

[5] V. Madani, D. Novosel, S. Horowitz, M. Adamiak, J. Amantegui, D. Karlsson, S. Imai, A. Apostolov, IEEE PSRC report on global industry experiences with system integrity protection schemes (sips), IEEE Trans. Power Del. 25 (2010) 2143-2155.

[6] A. G. Phadke, J. S. Thorp, Synchronized Phasor Measurements and their Applications, Springer, New York NY, 2008.

[7] A. Phadke, R. M. de Moraes, The wide world of wide-area measurement, IEEE Power and Energy Mag. 6 (2010) 52-65.

[8] J. Momoh, Smart Grid: Fundamentals of design and analysis, WileyIEEE Press, Hoboken NJ, 2012.

[9] A. P. S. Meliopoulos, G. Cokkinides, R. Huang, E. Farantatos, S. Choi, Y. Lee, X. Yu, Smart grid technologies for autonomous operation and control, IEEE Trans. Smart Grid 2 (2011) 1-10.

[10] C. W. Taylor, Power System Voltage Stability, EPRI Power System Engineering Series, McGraw Hill, New York, 1994.
[11] C. Vournas, G. Manos, Emergency tap-blocking to prevent voltage collapse, Proc. 2001 IEEE PES PowerTech Conference, Porto, Portugal.

[12] C. Vournas, M. Karystianos, Load tap changers in emergency and preventive voltage stability control, IEEE Trans. on Power Systems 19 (Feb. 2004) 492-498.

[13] B. Otomega, V. Sermanson, T. Van Cutsem, Reverse-logic control of load tap changers in emergency voltage conditions, Proc. 2003 IEEE PES PowerTech Conference, Bologna, Italy.

[14] C. W. Taylor, Concepts of undervoltage load shedding for voltage stability, IEEE Trans. Power Del. 7 (1992) 480-487.

[15] B. Ingelsson, P. Lindstrom, D. Karlsson, G. Ruvnik, J. Sjodin, Special protection scheme against voltage collapse in the South part of the Swedish grid, Proc. CIGRE Conf.

[16] Z. Feng, V. Ajjarapu, D. J. Maratukulam, A practical minimum load shedding strategy to mitigate voltage collapse, IEEE Trans. Power Syst. 13 (1998) 1285-1291.

[17] C. Moors, D. Lefebvre, T. Van Cutsem, Load shedding controllers against voltage instability: a comparison of designs, Proc. 2001 IEEE PES PowerTech Conference, Porto, Portugal.

[18] V. C. Nikolaidis, C. D. Vournas, Design strategies for load shedding schemes against voltage collapse in the hellenic system, IEEE Trans. Power Syst. 23 (2008) 582-591.

[19] C. Vournas, A. Metsiou, M. Kotlida, V. Nikolaidis, M. Karystianos, Comparison and combination of emergency control methods for voltage stability, Proc. IEEE PES 2004 General Meeting, Denver, USA.

[20] T. Van Cutsem, C. D. Vournas, Emergency voltage stability controls: An overview, Proc. IEEE PES 2007 General Meeting, Tampa, USA.

[21] F. Capitanescu, B. Otomega, H. Lefebvre, V. Sermanson, T. Van Cutsem, Decentralized tap changer blocking and load shedding against voltage instability: Prospective tests on the rte system, Int. Journal Elec. Power and Energy Syst. 31 (2009) 570-576.

[22] B. Otomega, T. Van Cutsem, Undervoltage load shedding using distributed controllers, IEEE Trans. Power Syst. 22 (2007) 1898-1907.

[23] M. Glavic, T. Van Cutsem, Wide-area detection of voltage instability from synchronized phasor measurements. Part I: Principle. Part II: Simulation results, IEEE Trans. Power Syst. 24 (2009) 1408-1425.

[24] M. Glavic, T. Van Cutsem, Reconstructing and tracking network state from a limited number of synchrophasor measurements, IEEE Trans. Power Syst. 28 (2013) 1921-1929.

[25] P. Kundur, Power System Stability and Control, The EPRI power system engineering series, McGraw-Hill, 1994.

[26] B. Otomega, T. Van Cutsem, A load shedding scheme against both shortand long-term voltage instabilities in the presence of induction motors, Proc. 2011 IEEE PES PowerTech Conference, Trondheim, Norway. 